\title{
Spherical mapping of a perforated-wall model
}

\author{
Mike Christenson $^{1 *}$, AIA, and Malini Foobalan ${ }^{2}$
}

\author{
${ }^{1}$ Assistant Professor of Architecture, North Dakota State University, Fargo, North Dakota (USA), 612-209-0764 \\ mike.christenson@ndsu.edu, *corresponding author. \\ ${ }^{2}$ Graduate Research Assistant and M. Arch. Candidate, North Dakota State University, Fargo, North Dakota \\ (USA), malini.foobalan@my.ndsu.edu
}

doi:10.5618/arch.2012.v1.n1.2 || Received: 22-1-2012, Accepted: 27-1-2012, Available online: 31-1-2012

\begin{abstract}
This essay discusses the general problem in visibility analysis of mapping a building envelope, i. e., the physical membrane separating a building's interior from its exterior, onto a hollow sphere. A digital model of the building envelope is constructed as a perforated-wall model and a constructive method is introduced to produce a spherical map of the model. The construction of spherical maps provides a means of comparison between buildings and environments, and between vantage points within buildings. Possible future approaches are suggested here, including the superimposition of one spherical map onto another to test for similarities and differences. The Alcuin Library at St. John's University in rural Minnesota (USA), designed by architect Marcel Breuer, is used as a test case for the ideas introduced in this paper.
\end{abstract}

Keywords: Isovist; visibility analysis; occlusion; Breuer, Marcel; digital modeling

\section{Introduction}

Visibility analysis, which can be broadly defined as the study of what pedestrians can see within an environment $\mathrm{E}$, is often carried out in urban environments or in landscapes as a means of mapping quantifiable aspects of visual perception, usually with the aim of demonstrating relationships between visual perception and human cognition and behavior [1]. Within the general scope of visibility analysis, occlusion is a critically important issue. In the context of visibility analysis, occlusion refers to those features within $\mathrm{E}$ which occlude a pedestrian's view of a hypothetical distant horizon. For example, a free-standing building viewed from its exterior at ground level occludes the view of the horizon beyond. Meanwhile, the same building occludes views of the horizon for persons within the building. Thus, occlusion can be seen as a concern from both an exterior or "urban" point of view and an interior point of view (Fig. 1).
The isovist is a key component in visibility analysis. Generally speaking, the isovist is a representation of that portion of $\mathrm{E}$ visible from a station point, $\mathrm{P}$. Isovists, which can be considered in either two dimensions (in plan or section), or in three dimensions (as volumetric models), register occlusion within $\mathrm{E}$ by highlighting precisely those areas or volumes which remain visible and unoccluded as seen from P. Conventionally, isovists are drawn to represent visible areas or volumes with an apex at $\mathrm{P}$, projected to an arbitrary circle or sphere centered on P (Fig. 2).

The concept of isovist is usually credited to Tandy's work on recording visible features of landscapes [2], although Tandy acknowledges in his essay that Alex Hardy, of the University of Newcastle, actually initiated the idea. Tom Porter, in 1979, introduced a graphic device essentially identical to the isovist, which he called a spatial boundary diagram [3]. However, the seminal work concerning isovists in visibility analysis is generally considered to be a 1979 paper by Michael Benedikt, citing both Tandy and Hardy [4]. Benedikt's paper established core isovist concepts and vocabulary, including the important idea of isovist field, which proposes that important attributes of visible perception within $\mathrm{E}$ can be captured by summing the properties of multiple isovists deployed in a field. Critically, Benedikt in his 1979 paper identified the possibility of relating "kinds of space" - e. g., hall, court, and plaza - to isovists and isovist fields, suggesting that isovists constituted a viable form of inquiry from both the "urban" and "exterior" points of view. Although Benedikt's methods for visibility analysis remained limited to 2D isovists, he suggested the importance of threedimensional representations as a way of registering movement in E over time. For this purpose, Benedikt discussed the "Minkowski model" or isovist stack, a physical model constructed of 2D isovists stacked atop each other, arranged in an order corresponding to a movement path through E. 

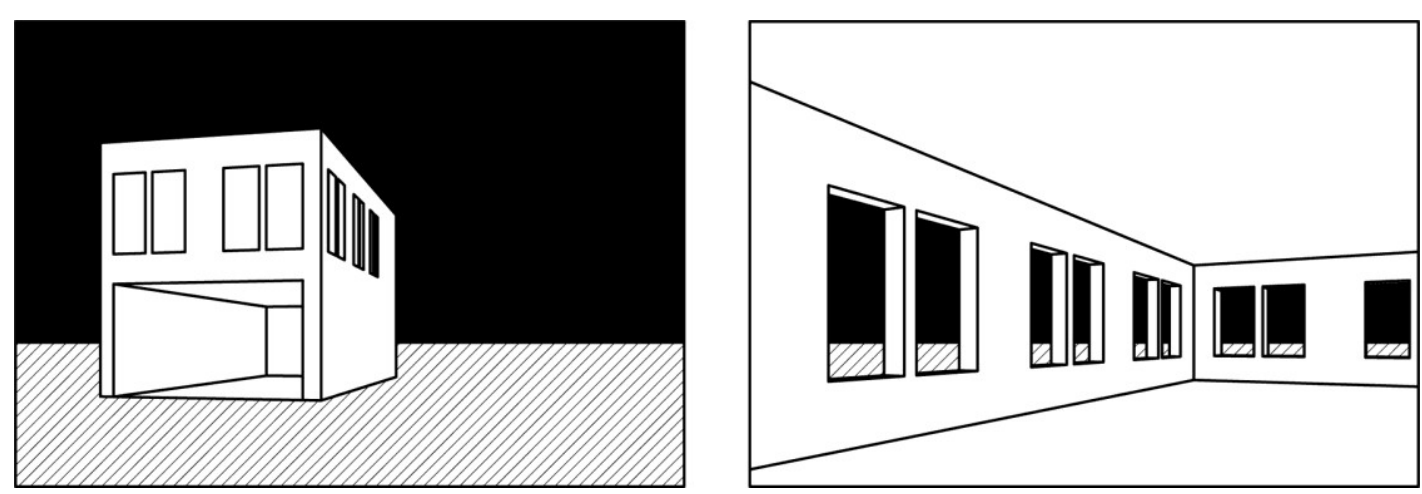

Figure 1. Occlusion in E, seen from a building's exterior (left) and interior (right).
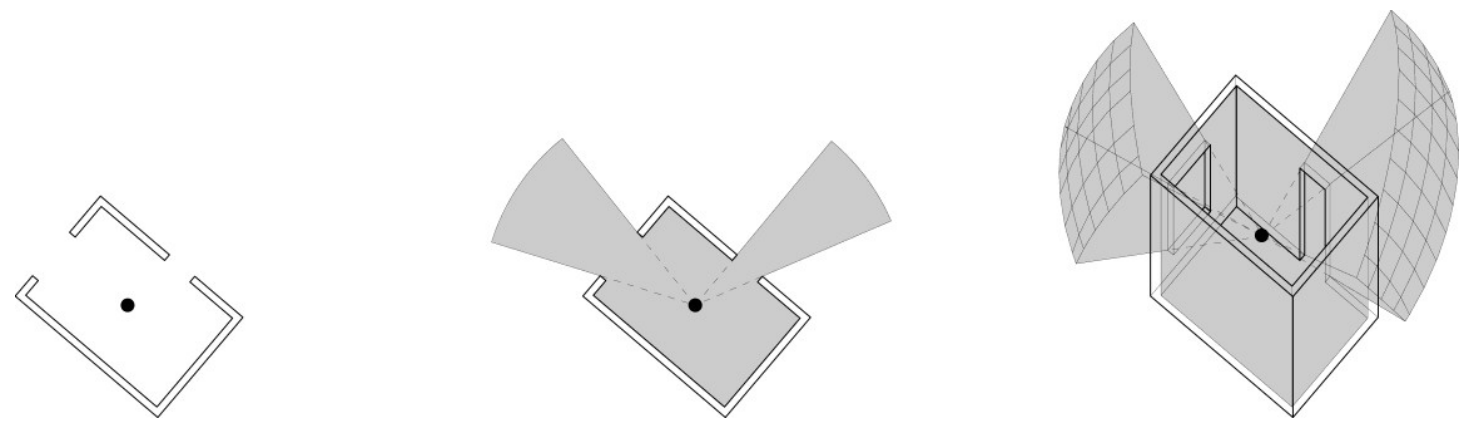

Figure 2. Generation of $2 \mathrm{D}$ and $3 \mathrm{D}$ isovists from a subset of $\mathrm{E}$.

Three papers written in the 1990s represent important contributions to visibility analysis applied to building interiors. Julienne Hanson, in a 1994 paper which set out to identify "design prestructures" within a selection of architect-designed houses, departed from the focus on isovist fields advocated by Benedikt and others [5]. Instead, Hanson chose to construct isovists within floor plans of existing buildings, basing her conclusions in part on the observable changes in isovist shape as the station point $\mathrm{P}$ moved within E. Turner and Penn, in a 1999 paper, considered the importance of relational ("syntactic") linkages between isovists within E, using London's Tate Gallery as a testing ground [6]. Their choice of the Tate derived from an earlier technical report prepared on the same building by Bill Hillier; Hillier's report made it possible for Turner and Penn to compare their results to already-completed studies of visitor movement within the Tate's rooms. A third paper, by Peponis et al, in noting that all but the simplest building interiors are not perceived in their entirety at once, proposed methods for identifying and analyzing visually perceptible transitions between discretely partitioned interior spaces [7].

Two significant 2003 papers articulated distinct approaches for 3D visibility analysis, although both papers chose to focus on applications for exterior spaces in urban environments. Teller [8] utilized a 2D map of a spherical projection, which enabled him to generate a metric for sky opening, representing the percentage of hemispherical sky dome visible from a point within E. Teller demonstrated the application of his method to situations involving the design of new buildings in an urban environment. Contemporaneously with Teller, Fisher-Gewirtzman and Wagner [9] proposed a metric based on solid angles within a spherical model. Their Spatial Openness Index or SOI registered the volume of open space visible from a fixed point in E. They demonstrated the relevance of SOI as a basis for comparative evaluations of models. Like Teller, their work was focused on exterior spaces between buildings, and their demonstration considered a situation similar to that used by Teller (i. e., the measurable implications of constructing one or more new "obscuring buildings" within E). Their analysis more so than Teller's - was akin to Benedikt's work with isovists; their methodology bears procedural similarity to ours as discussed in this paper.

Three comparatively recent papers suggest the continuing viability of visibility analysis as a fruitful area of research. Yang, Putra, and Li, in proposing "Viewsphere analysis," demonstrated the significance of z-dimension in constructing 3D isovists [1]. Morello and Ratti [10] considered 3D isovists as part of their approach to reconsidering the seminal urban analyses of Kevin Lynch. Finally, Van Bilsen [11] argued the 
importance of 3D visibility analysis and considered the construction of 3D isovists in his discussion of "serious games." However, despite the continuing viability of visibility analysis research, the extensive work in urban applications extending over thirty years since Benedikt's earliest papers has resulted in a situation where the problem of $3 \mathrm{D}$ visibility analysis from the point of view of building interiors remains understudied. Of course much important work has addressed interiors, including some work previously mentioned here $[5,6,7]$. A recent paper by Fisher-Gewirtzman [12] acknowledges the deficiency in interior-focused visibility analysis work and begins to establish an approach to integrating visibility analysis of interior spaces with urban considerations.

In this essay, we aim to further develop the question of visibility analysis within interior spaces of buildings in E. Our method considers the device of the perforated-wall model, that is, a model of a continuous building envelope perforated by openings. Consider as the general situation of a perforated-wall model a hollow regular cube with two openings (Fig. 2), representing a proportionately-scaled model of a subset of E, e. g., a one-room building placed in an open field. More complex cases can be derived from this general case, such as subsets involving multiple openings, multiple enclosures, non-rectilinear spaces, changes in floor or ceiling level, and internally obscuring features such as columns. The question arises whether any subset of E (e. g., a city, neighborhood, building, apartment, or room) can be effectively modeled as a perforated-wall model. Initial investigation of this question suggests that there are no obvious technical difficulties to constructing perforated-wall models of any given subset of E, but that the application of the modeling technique is not neutral with respect to the conclusions that can be drawn concerning visibility analysis.
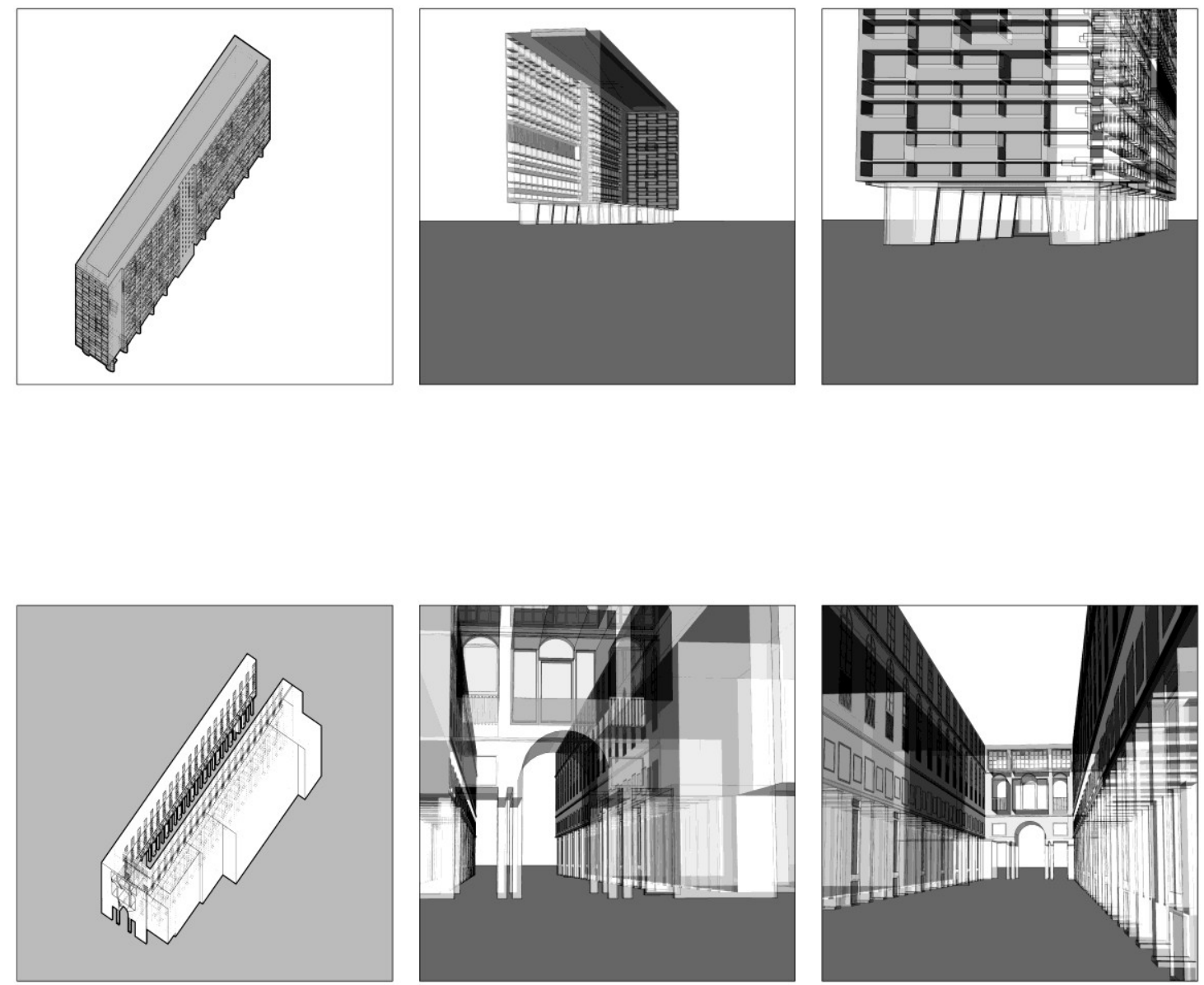

[Figure 3: Uffizi and Marseilles]

For example, consider a comparison between the Portico degli Uffizi in Florence, Italy, and the Unite d'Habitation in Marseilles, France (Figure 3). Schumacher [13] notes that "the Unite is a rectangular prism, oblong and solid. The Uffizi is a rectangular prism, oblong and void. ... [E] ach represents a way of looking at the city." This observation parallels the distinction between the "urban" and "interior" points of 
view with respect to the issue of occlusion. Generally speaking the Portico degli Uffizi is enclosed on four sides (two long sides and two short ones) and open to the sky. Its two short sides can be understood as thick, inhabitable walls perforated by openings (windows and arcades). A perforated-wall model of the Portico degli Uffizi registers these attributes, and others, which can be expected to affect visual perception to persons within the space or outside it. The similarlyproportioned Unite d'Habitation can be generally understood as an opaque block, and yet, a perforatedwall model registers its fundamental condition of interiority. A comparison of the two models points to that attribute, common to any subset of $\mathrm{E}$, that buildings always operate like masks, both obscuring what is behind as well as providing constraints to vision of the city from within.

In the discussion which follows, the letter $\mathrm{W}$, with appropriate subscript, is used to indicate a perforatedwall model of a building within E. For a simple building exhibiting the volume characteristics of a rectangular prism, $\mathrm{W}$ can be modeled as a hollow box with a zero-thickness exterior wall, perforated at locations corresponding to windows in the reference building. Consider a hollow, thin-walled sphere $\mathrm{S}$, completely surrounding $\mathrm{W}$ and centered at a station point P. A spherical mapping of $\mathrm{W}$ is carried out by projecting lines of sight from $\mathrm{P}$ to their intersection with $\mathrm{S}$. This procedure is essentially similar to that of constructing pyramidal solid angles, much as described by Fisher-Gewirtzman and Wagner in their approach to defining SOI. For clarity, we will use the letter S to represent both the sphere itself, as well as the map of W projected onto $\mathrm{S}$.

As should be obvious, the configuration of $\mathrm{S}$ will vary according to the location of $\mathrm{P}$ within $\mathrm{W}$, just as a two-dimensional isovist will vary its shape as its station point moves with respect to a plan opening (Hanson 1994). Thus, a specific instance of $\mathrm{S}$ is always identified with a corresponding $\mathrm{P}$, that is, $\mathrm{S}_{\mathrm{P}}$. For more complex buildings, the configuration of $S_{P}$ will vary depending on features registered within $\mathrm{W}$ such as interior partitions and exterior walls of non-zero and non-uniform thickness. If a digital model $\mathrm{W}$ is constructed, a complete $S_{P}$ can be determined by repeatedly projecting lines of sight from $\mathrm{P}$ as described here.

\section{Materials and Methods}

Marcel Breuer's architecture - specifically, his design for the Alcuin Library in Collegeville, Minnesota (USA) - is a particularly apt test case for exploring questions of the perforated wall because of Breuer's abiding interest in perforated and modular facade compositions, particularly his repeated use of "flue tile" to construct screens.

St. John's University, located in a rural area near St. Cloud, Minnesota, between Fargo, North Dakota, and Minneapolis, Minnesota, commissioned Breuer in 1953 to design a comprehensive plan for the campus. Ultimately, Breuer designed several buildings within the plan, the most well-documented of which is the Abbey Church (Fig. 4). Construction on the Alcuin Library (Fig. 5), which is sited directly across a grassed square from the Abbey Church, was completed in 1966. The library is in the shape of a rectangular prism. Its upper level consists primarily of a single two-story volume, the main reading room, the concrete roof structure of which is supported by two large "tree columns" made of cast-in-place concrete. The reading room is lit by story-high clerestory windows which are set behind an exterior screen constructed of "flue tile" laid in a vertical running bond pattern (Fig. 6).

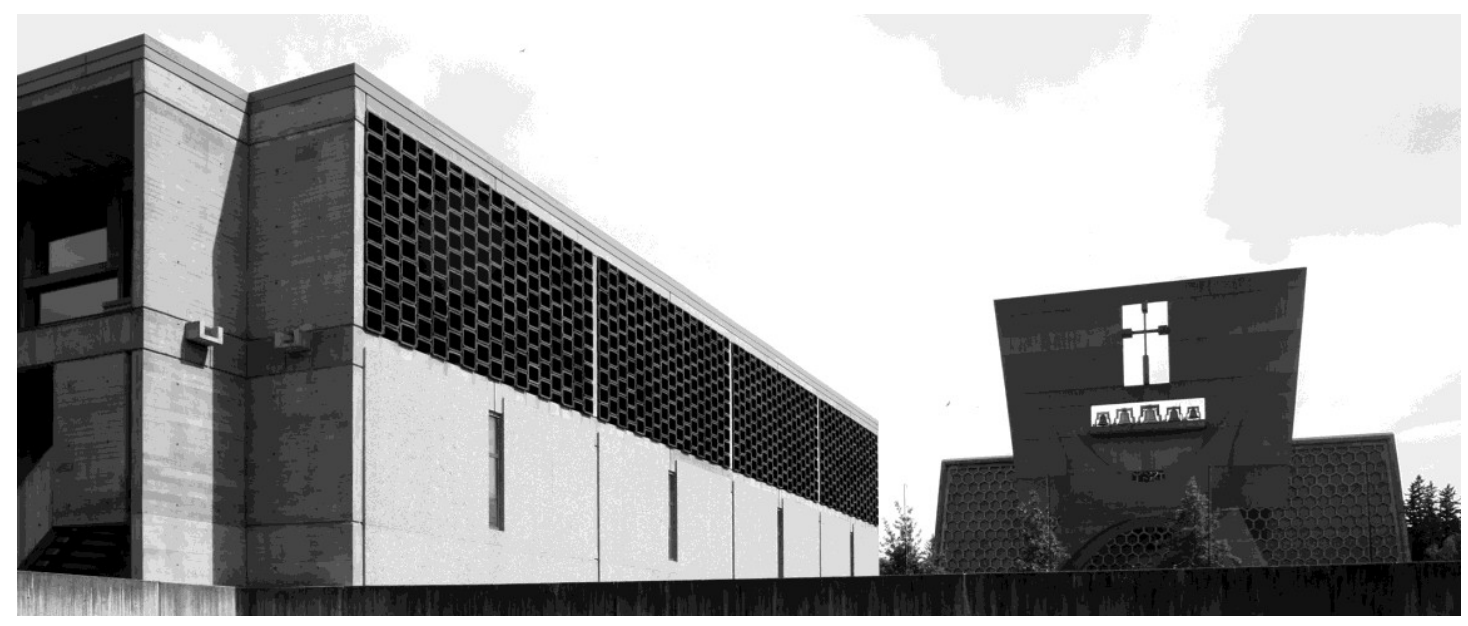

[Figure 4: Alcuin Library (left) and Abbey Church(right)] 


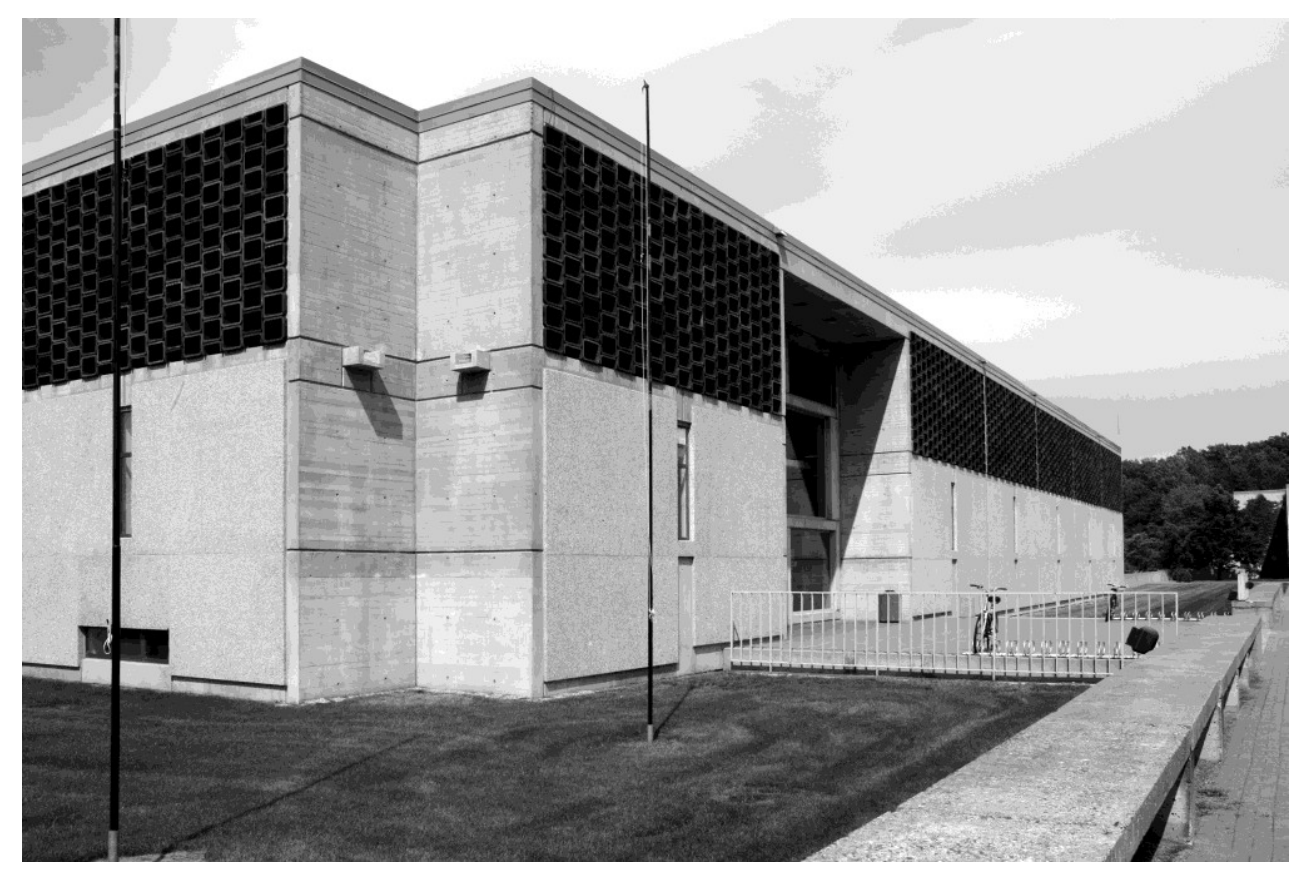

[Figure 5:

Alcuin Library]

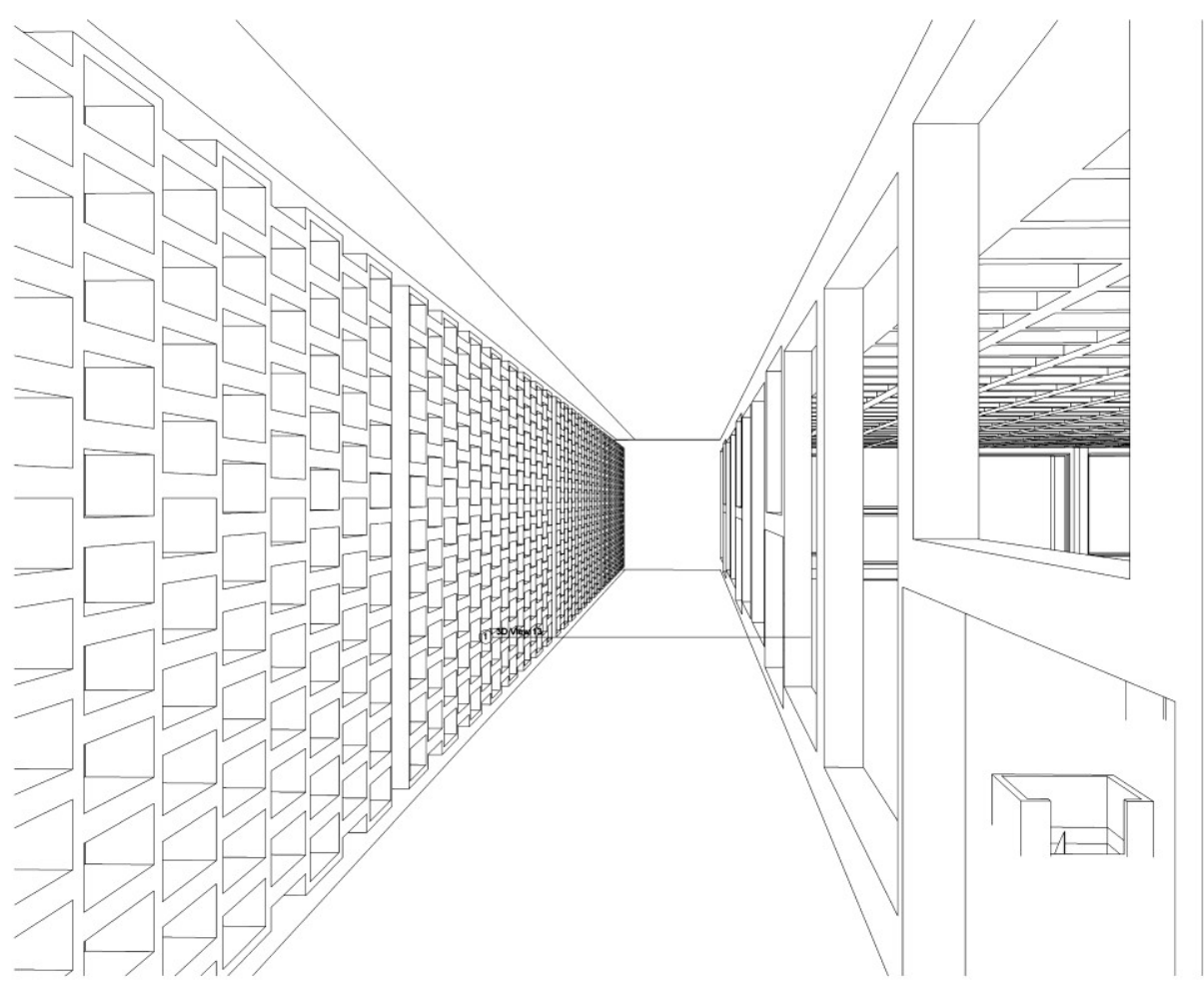

[Figure 6: flue tile at clerestory level, Alcuin Library.] 
Photographs of the building interior register the effect which the screens of flue tile have on visual perception (Figure 7). Because the flue tile screens are at the clerestory level, they generally filter views of the sky rather than views of adjacent buildings.

To construct a digital model of the Alcuin Library, we referred to original construction drawings stored in an on-site archive at St. John's University. We conducted field investigations of the existing building in an attempt to verify the accuracy of the construction drawings against the built structure. Our choice of Autodesk Revit as digital modeling software was motivated by Revit's parametric modeling capabilities; moreover, Revit's camera made it possible for us to inspect the model from multiple perspectives during construction, enabling us to test correspondence between the model and our field photographs.

After we had digitally modeled solid walls within the library, and set them at their correct heights, we modeled window openings. The library includes four different configurations of windows: two types on the east and west facades, and two types on the south facade. Therefore, in modeling the library in Revit, it made more sense to make a family for each window. However, because the windows repeat themselves on the grid, it was easier to build each bay as one family, and place them wherever applicable on the facade. This also ensured that the window locations were consistent throughout the model.

After modeling the windows, we proceeded to consider each facade separately, due to the fact that different details appeared on each facade. However, for the most part, the east and west facades share similar details, and hence, modeling commands could be repeated. Only toward the middle stages of model construction, as the model geometry became increaseingly complicated, were the capabilities and limitations of Revit seriously tested. The limitations of Revit became particularly clear with respect to the modeling of the flue tile, the concrete waffle slab at the reading room roof, and the tree columns.

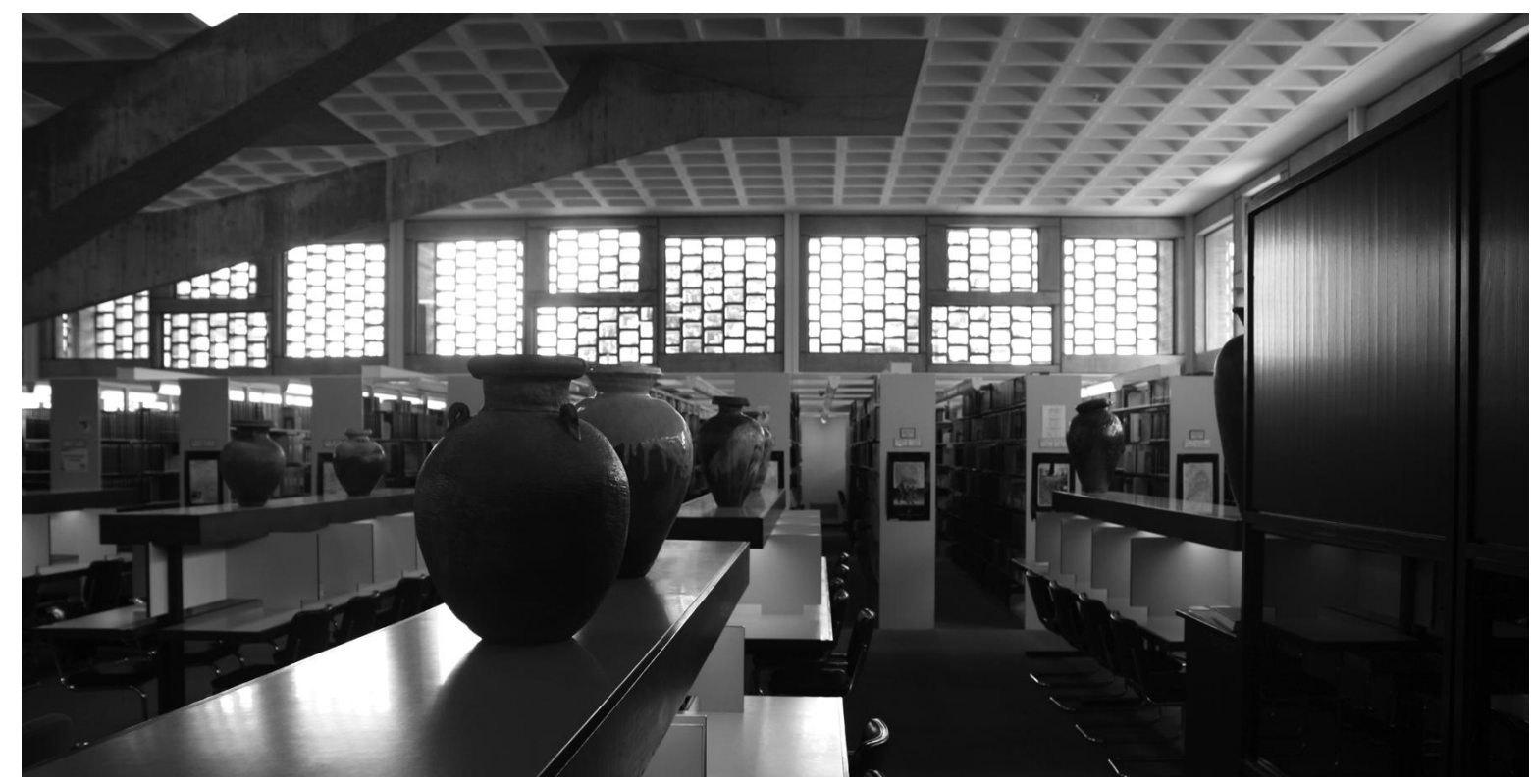

[Figure 7: Interior of Alcuin Library showing flue tile at clerestory level.]

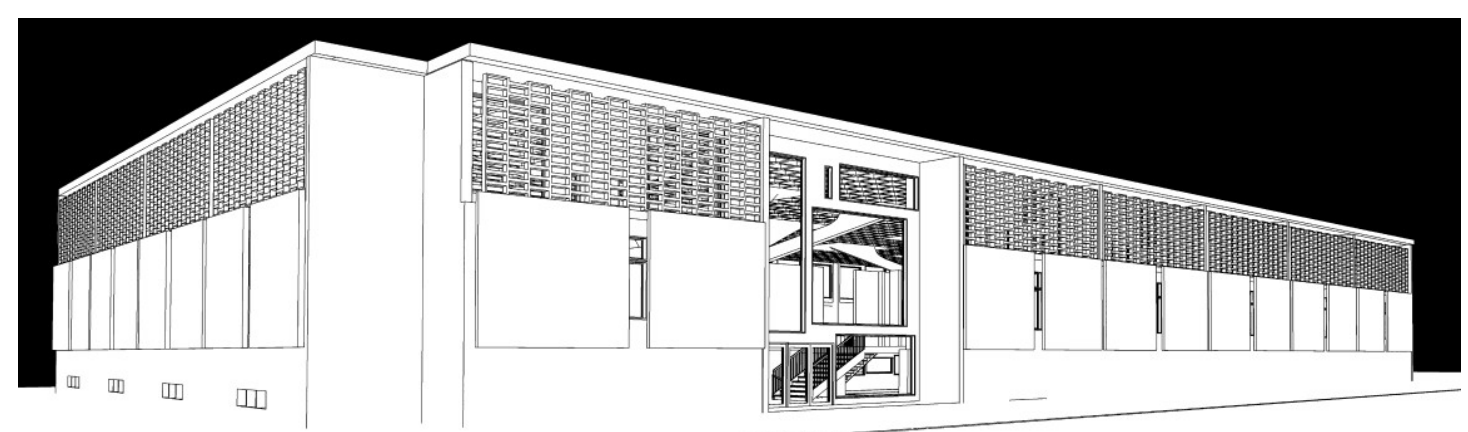

[Figure 8: Digital model of the Alcuin Library, viewed from front.] 


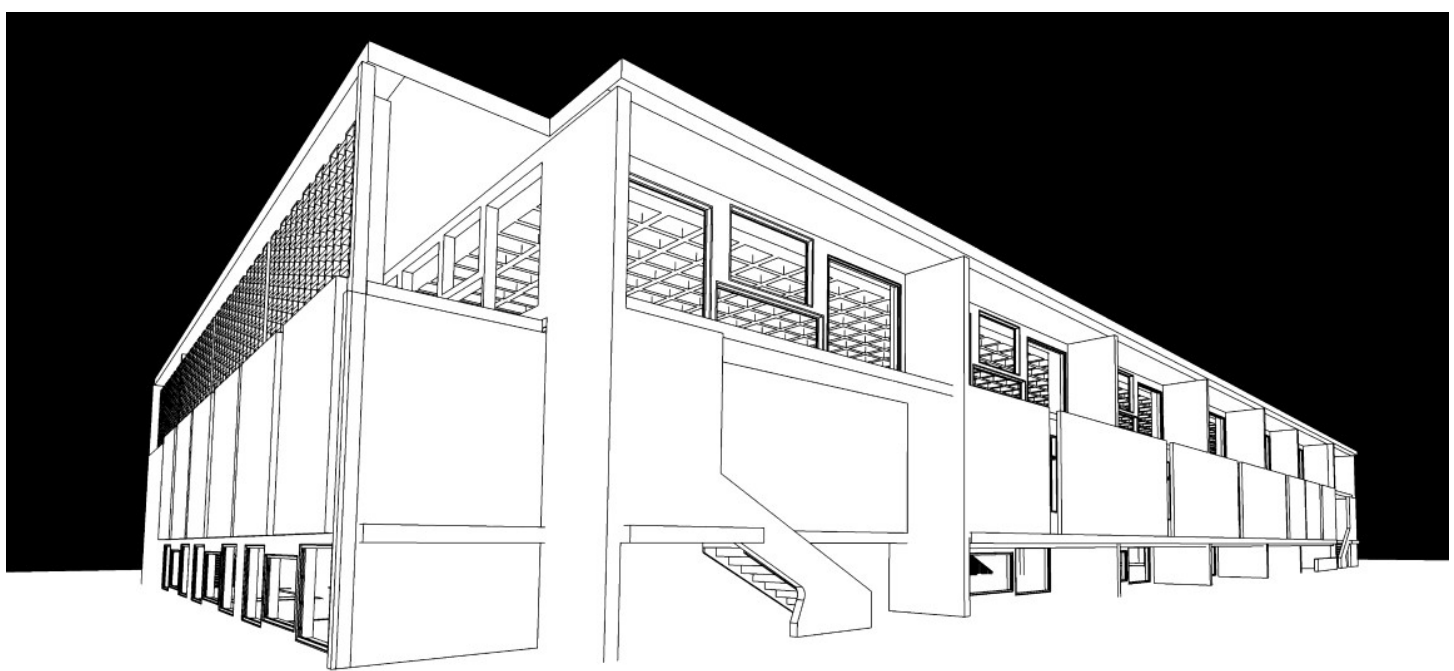

[Figure 9: Digital model of the Alcuin Library, viewed from back.]

The flue tiles (Fig. 6, 7), a common Breuer device, constitute at the Alcuin Library as an exterior screen at the reading room clerestory level. Because the tiles are laid in a vertical running bond pattern as they are made to fill rectangular openings, two different shapes of tile exist. The most prevalent shape is the full-sized tile, which constitutes the majority of the field. The second shape is the half-sized tile that sits alternately on the edges of the rectangular openings. We relied again on the original construction drawings to calculate the size of the flue tiles.
Each tile was initially modeled as a separate Revit family. However, the flue tile geometry, due to its detail and complexity, ultimately slowed the Revit model regeneration process to half-speed. We placed the flue tiles in panels to ease parameterization. In the resulting model, the tile panels are capable of being replaced in the future with new assemblies. Such replacement may further improve model performance, but more critically, we expect it to support further research into Breuer's use of the flue tile to modulate visibility.

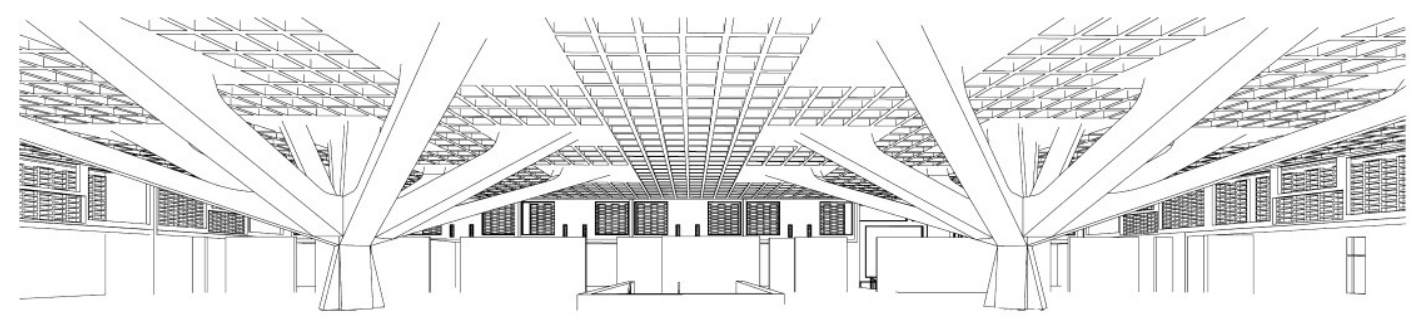

[Figure 10: tree column]

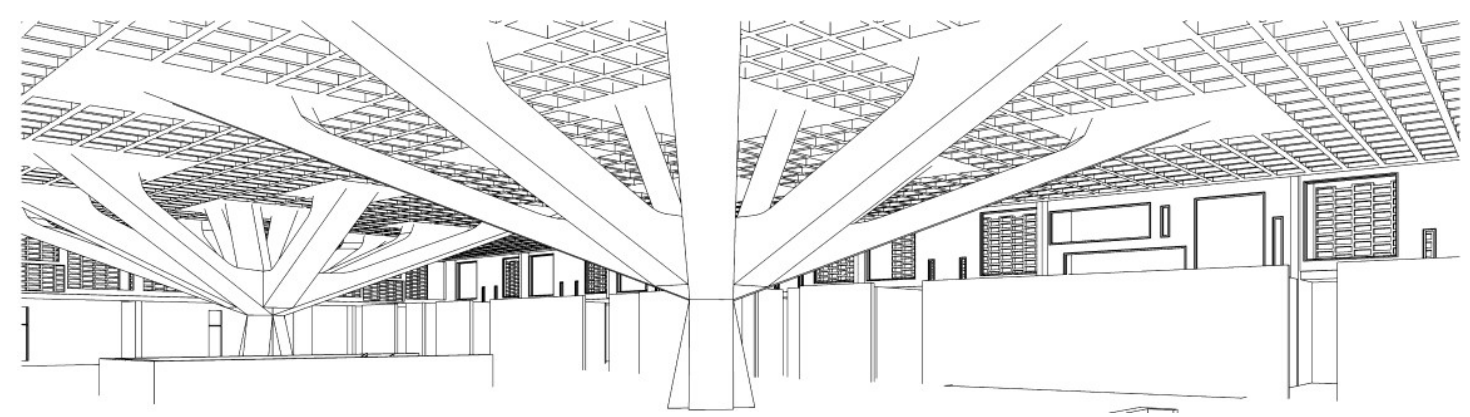

[Figure 11: tree column] 
The complexity of digitally modeling other building features, such as the concrete waffle slab and the tree columns, resulted not only from the geometry of the features but from the need to coordinate them with each other. In particular, the branches on the tree columns needed to align with corresponding points within the waffle slab. There were many size issues and alignment problems, which, through many iterations, were ultimately resolved in a manner corresponding to that shown in the original construction drawings.

For our initial tests, we ported the Revit model to AutoCAD, to take advantage of AutoCAD's simple suite of solid modeling tools. We simplified the library model by removing the flue tile and generalizing the clerestory windows to a single opening on each facade. We considered three different locations for $\mathrm{P}$ within $\mathrm{W}$, corresponding to three simulated viewing positions from the floor of the main reading room. For each $\mathrm{P}$, we constructed a unique $S_{P}$ by the following proce- dure:

1. Construct a 3D solid (an asymmetrical pyramid) using $\mathrm{P}$ as the apex and the visible corners of openings in the perforated-wall model as base vertices.

2. Extend the pyramid outward (away from $\mathrm{P}$ ) until it intersects $\mathrm{S}$.

3. Perforate $\mathrm{S}$ using a Boolean subtract operation.

Figure 12 shows the library model surrounded by $\mathrm{S}$, centered on $\mathrm{P}$, with a 3D solid extended outwards from $\mathrm{P}$ through an opening in the perforated wall model. Figure 13 shows several similar operations carried out in sequence. The result is a perforated sphere model, centered on $\mathrm{P}$.

From the point of view of $\mathrm{P}$, each model represents a perforated-wall model equivalent in its visibilityobscuring effect to the original perforated-wall model.

\section{Discussion}

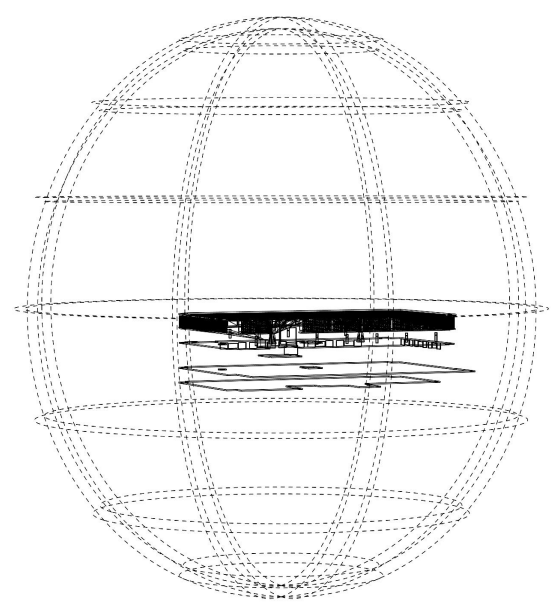

For any $\mathrm{P}$ within $\mathrm{W}$, the procedure described above projects a specific configuration of existing-building openings onto $\mathrm{S}$, resulting in a new set of openings, the configuration of which will vary according to the unique position of $\mathrm{P}$. (For this reason, we identify $\mathrm{S}_{\mathrm{P}}$ uniquely for each P.) If the building model is erased from the modeling environment (i. e., if $\mathrm{W}$ is subtracted from $E), S_{P}$ necessarily maintains the same constraints on visibility as $\mathrm{W}$, provided $\mathrm{S}_{\mathrm{P}}$ is viewed from $\mathrm{P}$. This follows from the way in which openings in $S_{P}$ are generated. The wall thickness of $\mathrm{S}$ has no effect on this result.

\section{Conclusions}

In this paper, we introduced a method for producing spherical representations of perforated-wall models as a means of mapping the envelope of a building in E. Although the test models constituted simplified representtations of a complicated building, the methodology is appropriate for structures of greater complexity.

Future work will address the question of comparison between different buildings and other subsets of E. For example, animations could be generated showing the changes in $\mathrm{S}_{\mathrm{P}}$ as $\mathrm{P}$ moves within $\mathrm{W}$. Such a representation would share with the Minkowski model discussed by Benedikt (Benedikt 1979) the characteristic that time and movement would be represented within a spatially static model. We also intend to superimpose multiple models to test correspondence of their structures of visibility.

Technical questions remaining to be addressed include the task of automating both the production of individual $\mathrm{S}_{\mathrm{P}}$ representations, as well as producing animated representations of multiple $S_{P}$ representations aggregated frame-by-frame to register time and movement within $\mathrm{E}$.

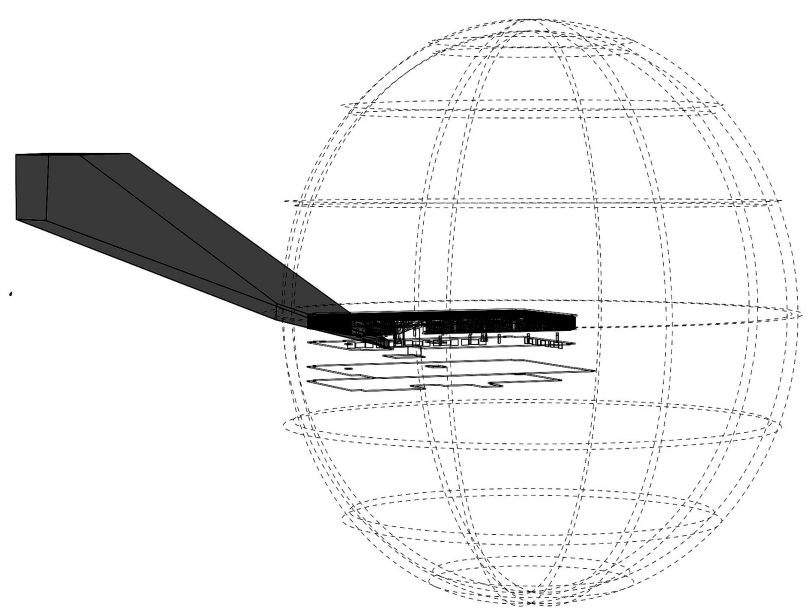

[Figure 12: sphere model] 

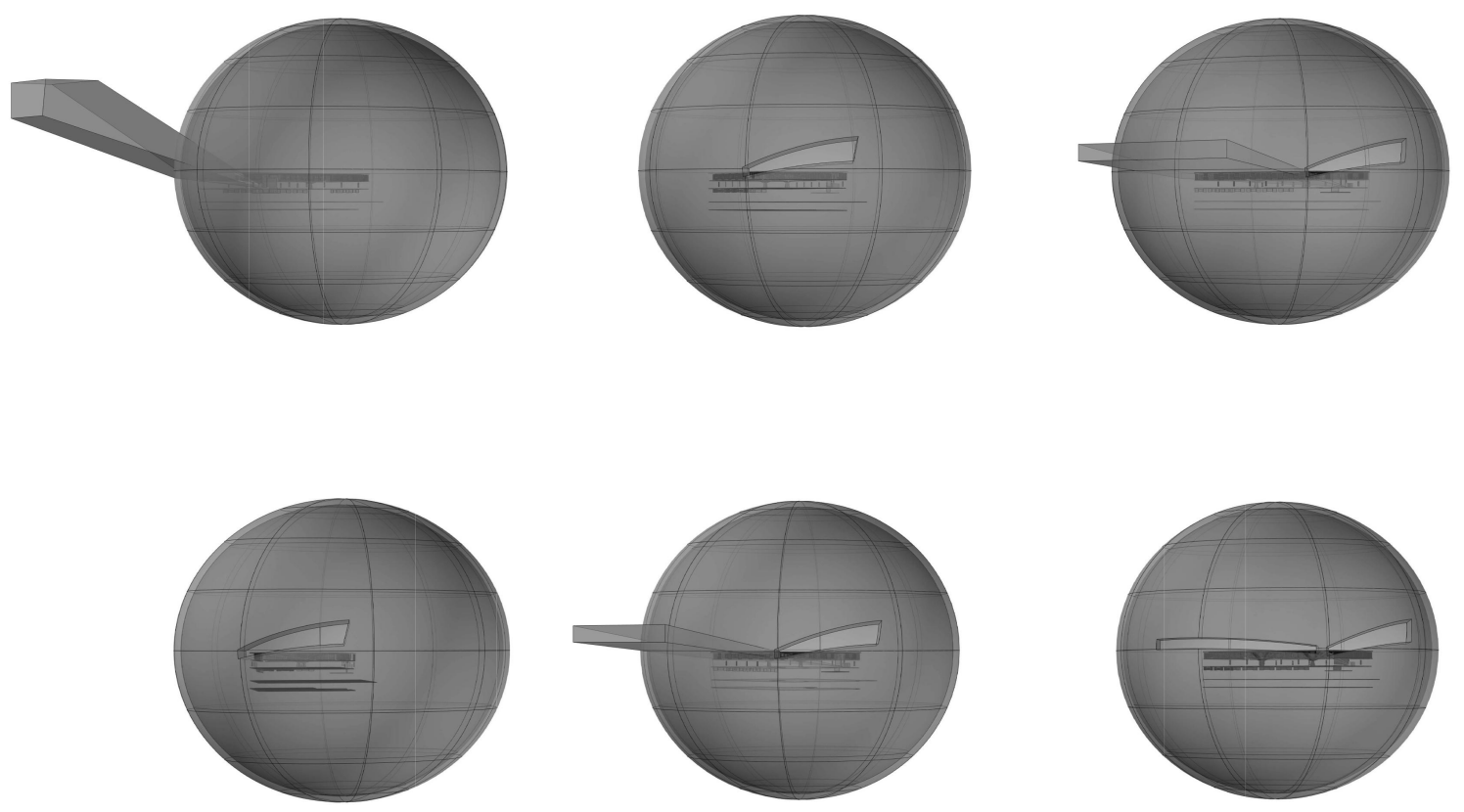

[Figure 13: sphere model]

\section{Acknowledgements}

Research for this paper was supported by a Graduate Research Assistantship funded by the Department of Architecture and Landscape Architecture at North Dakota State University. Illustrations in this paper, including photographs, are the work of the authors.

\section{References}

[1] Yang, P. P.-J.; Putra, S. Y.; Li, W. Environment and Planning B: Planning and Design 2007, 34, 971-992. "Viewsphere: a GIS-based 3D visibility analysis for urban design evaluation."

[2] Tandy, C. R. V., Symposium: Methods of Landscape Analysis, Landscape Research Group (London), 1967, 9-10. "The isovist method of landscape survey."

[3] Porter, T., How Architects Visualize, Van Nostrand Reinhold (New York), 1979.

[4] Benedikt, M., Environment and Planning B: Planning and Design 1979, 6, 47-65. "To take hold of space: isovists and isovist fields."

[5] Hanson, J. Environment and Planning B: Planning and Design 1994, 21, 675-704. "“Deconstructing' architects' houses."

[6] Turner, A.; Penn, A., Proceedings, 2nd International Symposium on Space Syntax, Universidad de Brasil, Brazil, 1999, 01.1-01.9. "Making isovists syntactic: isovist integration analysis."
[7] Peponis, J.; Wineman, J.; Rashid, M.; Kim, S. H.; Bafna, S., Environment and Planning B: Planning and Design 1997, 24, 761-781. "On the description of shape and spatial configuration inside buildings: convex partitions and their local properties."

[8] Teller, J., Environment and Planning B: Planning and Design 2003, 30, 339-356. "A spherical metric for the field-oriented analysis of complex urban open spaces."

[9] Fisher-Gewirtzman, D.; Wagner, I. A., Environment and Planning B: Planning and Design 2003, 30, 37-49. "Spatial openness as a practical metric for evaluating built-up environments."

[10] Morello, E.; Ratti, C., Environment and Planning B: Planning and Design 2009, 36, 837-853. "A digital image of the city: 3D isovists in Lynch's urban analysis."

[11] Van Bilsen, A., Proceedings, International Simulation and Gaming Association 2009, n. pag. "How can serious games benefit from 3D visibility analysis?"

[12] Fisher-Gewirtzman, D., Int. Arch. Photogramm. Remote Sens. Spatial Inf. Sci., 2011, XXXVIII-4/C21, 67-72. "Considering internal space layout as a majore [sic] component of visual analysis for urban systems and a key to reinterpret urban structures."

[13] Schumacher, T., Casabella, 1971, 359-360, 79-86. "Contextualism: Urban Ideals + Deformations." 